\title{
Metallicity retained by covalent functionalization of graphene with phenyl groups
}

\author{
Peizhe Tang ${ }^{a b}$, Pengcheng $\mathrm{Chen}^{a}$, Jian $\mathrm{Wu}^{a}$, Feiyu \\ $\mathrm{Kang}^{c}$, Jia Li*c, Angel Rubio ${ }^{\dagger b}$, and Wenhui Duan ${ }^{\ddagger a d}$ \\ ${ }^{a}$ Department of Physics and State Key Laboratory of Low-Dimensional Quantum Physics, \\ Tsinghua University, Beijing 100084, People's Republic of China \\ ${ }^{b}$ Nano-Bio Spectroscopy group, Dpto. Fúsica de Materiales, Universidad del País Vasco, \\ Centro de Física de Materiales CSIC-UPV/EHU-MPC and DIPC, \\ Av. Tolosa 72, E-20018 San Sebastián, Spain \\ ${ }^{c}$ Engineering Laboratory for Functionalized Carbon Materials and \\ Key Laboratory of Thermal Management Engineering and Materials, \\ Graduate School at Shenzhen, Tsinghua University, \\ Shenzhen 518055, People's Republic of China \\ ${ }^{d}$ Institute for Advanced Study, Tsinghua University, \\ Beijing 100084, People's Republic of China
}

(Dated: May 20, 2013)

\footnotetext{
* E-mail: lijia@phys.tsinghua.edu.cn

$\dagger$ E-mail: angel.rubio@ehu.es

$\ddagger$ E-mail: dwh@phys.tsinghua.edu.cn
} 


\begin{abstract}
To resolve the controversy over the functionalization effect on the conductivity, we systematically investigate the structural and electronic properties of graphene covalently functionalized with phenyl groups. Using first-principles calculations combined with the model Hamiltonian analysis, we find that the structural stability, electronic and transport properties of the functionalized graphene are strongly dependent on the adsorption site of the phenyl groups. In detail, double-side functionalized graphene is energetically more favorable than single-side functionalized graphene, and more importantly, they exhibit an exotic non-magnetic metallic state and a magnetic semiconducting state, respectively. For covalently double-side functionalized graphene, two bands contributed by $\pi$ electrons of graphene cross at the Fermi level with the preserved electron-hole symmetry, and the Fermi velocity of carriers could be flexibly tuned by changing the coverage of the phenyl groups. These results provide an insight to the experimental observation [ACS Nano 2011, 5, 7945], interpreting the origin of the increase in the conductivity of graphene covalently functionalized with phenyl groups. Our work reveals the great potential of these materials in future nanoelectronics or sensors by controlling the attachment of phenyl groups.
\end{abstract}




\section{INTRODUCTION}

In recent years, graphene has received considerable attention from the scientific community for its potential use as building block in the future nanoelectronic devices, $[1-4]$ due to its high charge-carrier mobility,[5, 6] current-carrying capability,[7] and thermal conductivity.[8, 9] The lack of a band gap, however, has hampered the applications of graphene, and functionalization has been proposed as a mechanism to introduce a band gap in graphene and bring it into the realm of Si-based electronics. [2, 10] On the other hand, the large scale synthesis of graphene in a realistic condition, being extremely important for the real industry, remains a major challenge for the scientists.[11-13] Owing to the low cost and easy fabrication processes, chemical solvent-based exfoliation of graphite is regarded as a possible way to overcome this bottleneck.[14-17] During this process, the graphene could be covalently functionalized. Recently, considerable experimental works have focused on diazonium salts, which are the reactive reagents and have been used to modifying the surface of $s p^{2}$-hybridized carbon materials[18-21], epitaxial graphene [22, 23] and exfoliation graphene[24-28]. It is found that the graphene grafting by phenyl groups (specifically, 4-tert-butylphenyl groups, TBP)[27] can be achieved through effective reductive activation. Several spectroscopy techniques, for example Raman spectroscopy, atomic force microscopy and high-resolution transmission electron microscopy, have been utilized to identify the phenyl functional groups on the basal plane of graphene.[27]

From the theoretical viewpoint, the covalent functionalization of graphene will introduce local defects, leading to some new physics in the Dirac electron system.[29, 30] In the honeycomb lattice of graphene, there exist two equivalent sublattices (denoted as A and B). An isolated point modification on sublattice A can induce a quasi-localized virtual bound state (VBS) on the neighboring atoms belonging to sublattice B due to the breaking sublattice symmetry. The wave function of the VBS has three folded symmetry $\left(D_{3 h}\right)[29]$ and decays as $1 / r$ at large distances. [31] The exchange coupling between adjacent point defects can result in the splitting of VBS to induce ferromagnetism in graphene at certain concentrations of point defects.[32-35] The introduction of VBS in graphene normally can also open a band gap around the Fermi level; however, the Dirac cones in the graphene may be preserved, depending on the concentration and spatial arrangement of the chemisorbed groups used for chemical functionalization. $[36,37]$ 
In experimental situations, the conductivity of graphene with phenyl groups covalently bonded to its basal plane by different functionalization methods was observed to exhibit different behaviors (i.e., enhancement behavior [22, 38] and suppression behavior[26] induced by covalent functionalization), which is still under dispute. In addition, room temperature magnetic order has also been found in the phenyl-group functionalized graphene nanostructures. [39, 40] Therefore, in order to understand experimental observations of functionalized graphene, it is highly desirable to explore possible stable phenyl functionalized structures and the influence of functionalization on the electronic, transport and magnetic properties of graphene, which is helpful for its use in future nanoelectronics.

In this work, based on the first-principles calculations, we systematically investigate the structural and electronic properties of graphene covalently functionalized with TBP which is taken as a representative of the phenyl groups. We find that the single-side attachment of TBP on graphene (denoted as s-CFG) can induce a quasi-localized VBS, resulting in the ferromagnetism and gap opening as expected. On the other hand, single-side functionalization can activate the nearest-neighboring carbon atoms next to adsorption site, and consequently another TBP group can easily adsorb at the activated site on the opposite side to form more stable structure. Since no breaking of charge conjugation and space-time inversion symmetry occurs, covalently double-side functionalized graphene with TBP (denoted as dCFG) exhibits an exotic non-magnetic metallic state with two bands crossing at the Fermi level and the preserved electron-hole symmetry. Moreover, the Fermi velocity of carriers is sensitively dependent on the coverage of the phenyl groups. Because the metallic bands are mainly contributed by $\pi$ electrons of graphene but have little connection with the detailed structure of the phenyl groups, it is anticipated that the metallic state can be present in graphene functionalized with other phenyl groups, such as nitrophenyl groups. Importantly, our two dimensional (2D) quantum transport calculations clearly reveal that s-CFG shows a semiconductor behavior, whereas d-CFG a metallic transport behavior. This can offer an insight towards resolving the controversy of recent experimental transport measurements of graphene functionalized with phenyl groups, and suggest that functionalized graphene can be assembled into various electronic devices by controlling the adsorption of phenyl groups. 


\section{MODELS AND METHODS}

The spin-polarized DFT calculations are performed via the Vienna ab initio simulation package (VASP)[41]. The projector augmented wave (PAW) potential[42] and the generalized gradient approximation with Perdew-Burke-Ernzerhof functional[43] are used to describe the core electrons and the exchange-correlation energy, respectively. The cutoff energy for the plane wave basis set is set to $450 \mathrm{eV}$. The Brillouin zone is sampled by Monkhorst-Pack $k$ points. The energies are converged to below $10^{-5} \mathrm{eV}$ and the residual forces on all atoms are converged to below $0.01 \mathrm{eV} / \AA$. A supercell with a vacuum distance of $12 \AA$ normal to the graphene plane is used. The accuracy of our method is benchmarked against a recent study[44], where the DFT calculations was used to determine the adsorption energy for the attachment of hydrogen atom on graphene with the surface coverage of $0.20 \mathrm{nmol} / \mathrm{cm}^{2}$. Our result $(-0.77 \mathrm{eV})$ is as the same as their result. The two-dimensional quantum transport properties are adopted via Atomic ToolKit package[45], which combines non-equilibrium Green's functions (NEGF) methods with DFT to simulate open systems in a two-probe geometry. Single- $\zeta$ polarized atomic basis sets for expanding the electronic density, the local density approximation for exchange-correlation energy, and converged $k$ meshes for the periodic boundary condition perpendicular to the transport direction are all well tested and adopted in the whole calculations.

\section{RESULTS AND DISCUSSION}

We start by investigating the geometrical structures of s-CFG and find that the interaction between the TBP group and the graphene sheet is quite weak in comparison with that between hydrogen/fluorine and the graphene sheet.[44, 46] The adsorption energy $\left(E_{\text {ad }}\right)$ of attaching one TBP group on graphene is just $-0.29 \mathrm{eV}$ and the zero point energy correction[47] is $11.37 \mathrm{meV}$. The binding energy is very close to that of isolated $\mathrm{C}_{6} \mathrm{H}_{5}$ radical adsorbed on graphene $(-0.25 \mathrm{eV})[48]$, but the resulting vibrational energy is smaller than that of the oxygen adsorpted on metal surface[47]. The weak interaction indicates that s-CFG is not very stable and the TBP group may be easily removed. In the optimized structure, as shown in Figure 1a, a $\mathrm{C}-\mathrm{C}$ bond of $1.59 \AA$ is formed between the TBP group and graphene sheet. Carbon atom A connected to the TBP group is displaced out of the basal plane of graphene 
sheet by $0.55 \AA$, causing a larger bond length between $\mathrm{A}$ and $\mathrm{B}$ atoms $(1.52 \AA)$ than that in the pristine graphene $(1.42 \AA)$. Correspondingly, the $s p^{3}$ hybridization occurs at the functionalized site, which induces a structural distortion of the graphene sheet. Decreasing the TBP coverage can release the local strain and thus lower the single-side adsorption energy significantly (Figure 2).

Since covalent functionalization of graphene with grafted TBP groups can be accomplished from solvent-based exfoliation of graphite, TBP groups may adsorb on both sides of graphene sheet. To investigate the double-side adsorption, we consider four adsorption sites on the opposite side of graphene sheet for attaching the second TBP group, as shown in Figure 3. It can be seen that among all possible binding sites for the second attachment, the ortho position of the graphene ring (Figure $1 \mathrm{~b}$ and Figure $3 \mathrm{a}$ ) is the most energetically favorable site: the adsorption energy $E_{\text {ad }}$ for attaching the second TBP group is $-0.93 \mathrm{eV}$, much lower than those of s-CFG $(-0.29 \mathrm{eV})$ and double $\mathrm{C}_{6} \mathrm{H}_{5}$ radical functionalized graphene sheet $(-0.64 \mathrm{eV})[48]$, indicating the double-side functionalized graphene with phenyl groups is most likely to form in the experiments[27]. When the second TBP group is attached at the para position (Figure 3c), the energy is $0.19 \mathrm{eV}$ higher than that of the most stable configuration (Figure 3a). In contrast, the meta position (Figure 3b) is the least stable with $E_{\text {ad }}$ of $-0.16 \mathrm{eV}$, even higher than that of s-CFG. The energy of the binding site beyond a hexagonal ring of the first attachment, as shown in Figure 3d, lies in between those of the ortho and meta positions (Figure $3 \mathrm{a}$ and Figure 3b). It is interesting to see that for the double side adsorption on the same hexagonal ring, the two TBP groups tend to bind to the carbon atoms belonging to different sublattices. This could be well understood by considering the electronic properties of s-CFG shown below. Moreover, the second attachment also enhances the interaction between all TBP groups and the graphene sheet, indicating that the groups can be hardly removed with double-side adsorption.

In the most energetically favorable d-CFG, which has the ortho configuration as shown in Figure 1b, the bond length between the TBP group and graphene sheet is $1.60 \AA$, very close to that of s-CFG $(1.59 \AA)$. And the lengths of bonds $A_{1}-B_{1}, A_{1}-B_{2}$ and $A_{2}-B_{1}$ (also marked in Figure 1b) are $1.57 \AA, 1.51 \AA$ and $1.51 \AA$ respectively. All of them are larger than C-C $s p^{2}$ bond length in pristine graphene $(1.42 \AA)$. Similar to s-CFG, the graphene carbon atoms $A_{1}$ and $B_{1}$ are displaced out of the basal plane of graphene sheet on opposite sides respectively. Thus, two $s p^{3}$ hybridizations are formed at the nearest-neighboring carbon 
site. On the other hand, in contrast to the s-CFG, the adsorption energy of d-CFG varies slightly with the coverage, as shown in Figure 2. This indicates that the formation of $s p^{3}$ bonds located on opposite sides of graphene sheet can effectively release the local strain in the vicinity of functionalized carbon sites.

More detailed understanding of the adsorption behavior could be obtained by analyzing the change of the electronic properties. Figures $4 \mathrm{a}$ and $\mathrm{b}$ show, respectively, the majorityspin and minority-spin band structures of s-CFG with the coverage of $0.20 \mathrm{nmol} / \mathrm{cm}^{2}$. Consistent with previous studies[29, 31, 33], quasi-localized VBSs (marked as $\alpha$ ) around the Fermi level are induced by the breaking of the sublattice symmetry, and consequently, the itinerant (Stoner) magnetism can be induced by the electron exchange interaction in this system. Evidently the splitting of VBS leads to a ferromagnetic ground state with magnetic moment of $1 \mu_{\mathrm{B}}$ per attachment site and these properties hardly change with the increase of coverage. This is in agreement with the recent experimental observations of room temperature magnetic order on the phenyl-group functionalized graphene nanostructures.[39, 40] Figure 4d shows the partial charge density distribution of $\alpha$ band at the $K$ point. Overall, the charge density of quasi-localized VBS state, which exhibits threefold symmetry $\left(D_{3 h}\right)$ and decays very quickly, is mainly concentrated at the graphene carbon atoms belonging to the sublattice different from that of functionalized carbon site. This charge distribution can activate the relevant graphene carbon atoms, to increase the adsorption energy of additional attached TBP groups on these sites. In Figure 2a, we plot the charge density difference of s-CFG. It can be seen that the $\sigma$ bond is formed between the TBP group and the graphene sheet. Moreover, the charge density around the attachment site is redistributed and the charge of carbon atoms B is more localized than that of pristine graphene. The distorted $s p^{2}$ hybridization of carbon atoms around the attachment site and the localization of charge on carbon atoms $\mathrm{B}$ render the ortho site as preferred adsorption site for the attachment of the second TBP group on opposite side.

Different from VBS in s-CFG, an exotic nonmagnetic metallic behavior is observed for d-CFG with the coverage of $0.20 \mathrm{nmol} / \mathrm{cm}^{2}$. As shown in Figure 4c, two dispersive bands (marked as $\beta$ and $\gamma$ ) with the width of $2 \mathrm{eV}$ cross each other at the Fermi level and the electron-hole symmetry is preserved, similar to the Dirac cone in the pristine graphene. However, the band-crossing point moves from the $K$ point to a nearby $k$ point and the Fermi velocity of carriers changes a lot compared with that of the pristine graphene. Toward better 
TABLE I: The Fermi velocity (in units of $10^{6} \mathrm{~m} / \mathrm{s}$ ) of carriers of d-CFGs with different coverages of TBP groups.

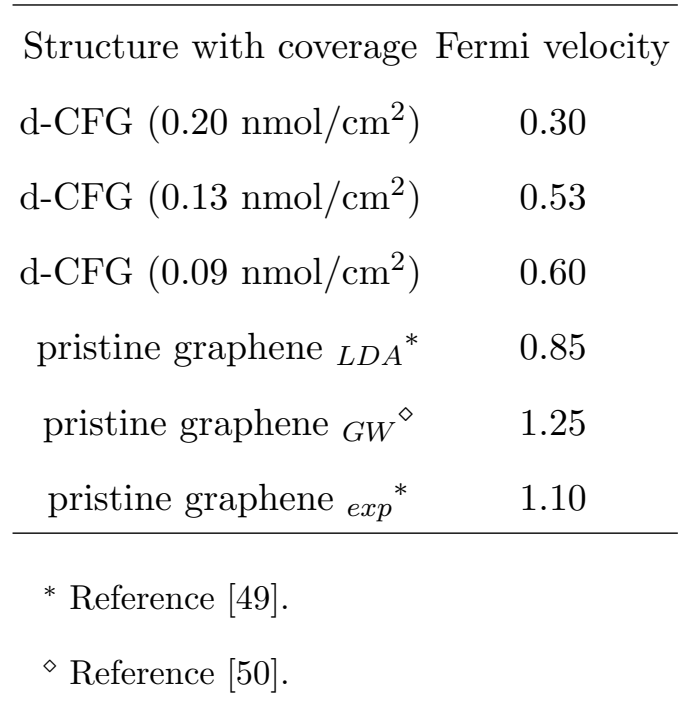

understanding of physical origin of the metallic bands, we plot the partial charge densities of $\beta$ and $\gamma$ bands at the $K$ point in Figure 4 d. It can be seen that the charge of $\beta$ and $\gamma$ bands, all contributed by $\pi$ electrons of graphene, distributes at the two equivalent sublattices with the twofold symmetry $\left(D_{2 h}\right)$. Especially, the distribution of charge density of the $\beta$ band is mainly along the armchair direction of graphene, while that of $\gamma$ band is along the zigzag direction (marked by the red arrows in Figure 4). Moreover, to clarify the relationship between the metallicity of d-CFGs and the coverage of TBP groups, we further calculate the band structures with the different coverage of TBP groups (from $0.09 \mathrm{nmol} / \mathrm{cm}^{2}$ to 0.20 $\left.\mathrm{nmol} / \mathrm{cm}^{2}\right)$. It is found that the d-CFGs with the different TBP coverages are metallic, but their detailed properties are highly dependent on the coverage of functionalization groups. As shown in Table 1, the Fermi velocity of carriers become larger with decreasing coverage of TBP groups and tends to approach the value of the pristine graphene. This indicates that one can significantly modify electrical conduction properties of d-CFG by changing the coverage of functionalization groups.

In order to gain further insight on the underlying mechanism of metallicity, a tightbinding Hamiltonian, with nearest-neighbor hopping of $p_{z}$ electron only, is used to describe d-CFG with the $2 \times 2$ supercell. In the model, two TBP groups are functionalized at two 
carbon sites $A_{1}$ and $B_{1}$ on opposite sides and the Hamiltonian can be written as

$$
H=-\gamma_{1}\left(a_{1}^{\dagger} b_{1}+H . c .\right)-\gamma_{2} \sum_{i>1}^{3}\left(a_{1}^{\dagger} b_{i}+H . c .\right)-\gamma_{2} \sum_{i>1}^{3}\left(a_{i}^{\dagger} b_{1}+H . c .\right)-\gamma_{0} \sum_{i>1}^{4}\left(a_{i}^{\dagger} b_{i}+H . c .\right)
$$

where $a_{i}$ and $b_{i}\left(a_{i}^{\dagger}\right.$ and $\left.b_{i}^{\dagger}\right)$ are annihilations (creations) of a $p_{z}$ electron on the two different sublattices, respectively. And $\gamma_{0}$ is the nearest-neighbor hopping energy in pristine graphene, $\gamma_{1}$ and $\gamma_{2}$ are the projection of hopping energy to the graphene plane between two attachment sites $\left(A_{1}\right.$ and $\left.B_{1}\right)$ and from attachment sites to the pristine sites, respectively. In this model, we neglect the energy difference of $p_{z}$ electron at different sites. As discussed above, the double-side attachment of two TBP groups results in $\gamma_{1} \gg \gamma_{0}$. Based on the perturbation theory[51], the effective Hamiltonian of $\pi$ electrons around the Fermi level $\left(H_{\text {eff }}\right)$ can be written as

$$
\left(\begin{array}{cccccc}
0 & f_{1}(\mathbf{k}) & 0 & f_{2}(\mathbf{k}) & 0 & f_{3}(\mathbf{k}) \\
f_{1}(\mathbf{k})^{\dagger} & 0 & f_{2}(\mathbf{k})^{\dagger} & 0 & f_{3}(\mathbf{k})^{\dagger} & 0 \\
0 & f_{2}(\mathbf{k}) & 0 & f_{1}(\mathbf{k}) & 0 & f_{4}(\mathbf{k}) \\
f_{2}(\mathbf{k})^{\dagger} & 0 & f_{1}(\mathbf{k})^{\dagger} & 0 & f_{4}(\mathbf{k})^{\dagger} & 0 \\
0 & f_{3}(\mathbf{k}) & 0 & f_{4}(\mathbf{k}) & 0 & f_{5}(\mathbf{k}) \\
f_{3}(\mathbf{k})^{\dagger} & 0 & f_{4}(\mathbf{k})^{\dagger} & 0 & f_{5}(\mathbf{k})^{\dagger} & 0
\end{array}\right)
$$

where $f_{1}(\mathbf{k})=-\gamma_{0} e^{i \boldsymbol{\delta}_{1} \cdot \mathbf{k}}+\gamma_{1}^{-1} \gamma_{2}^{2} e^{i\left(-\boldsymbol{\delta}_{1} \cdot \mathbf{k}+2 \boldsymbol{\delta}_{2} \cdot \mathbf{k}\right)}, f_{2}(\mathbf{k})=\gamma_{1}^{-1} \gamma_{2}^{2} e^{i\left(-\boldsymbol{\delta}_{1} \cdot \mathbf{k}+\boldsymbol{\delta}_{2} \cdot \mathbf{k}-\boldsymbol{\delta}_{3} \cdot \mathbf{k}\right)}, f_{3}(\mathbf{k})=$ $-\gamma_{0} e^{i \boldsymbol{\delta}_{3} \cdot \mathbf{k}}, f_{4}(\mathbf{k})=-\gamma_{0} e^{i \boldsymbol{\delta}_{2} \cdot \mathbf{k}}, f_{5}(\mathbf{k})=-\gamma_{0} e^{i \boldsymbol{\delta}_{1} \cdot \mathbf{k}}, \boldsymbol{\delta}_{1}=\left(\frac{a}{\sqrt{3}}, 0\right), \boldsymbol{\delta}_{2}=\left(-\frac{a}{2 \sqrt{3}},-\frac{a}{2}\right), \boldsymbol{\delta}_{3}=$ $\left(-\frac{a}{2 \sqrt{3}}, \frac{a}{2}\right)$, and $a$ is the lattice constant of pristine graphene. It is found that $H_{\text {eff }}$ satisfies charge conjugation symmetry, which implies $C H_{e f f} C^{\dagger}=-H_{\text {eff }}$, under the charge conjugation operation $C$ and $C^{\dagger}$. Thus the band structure of the d-CFG exhibits electron-hole symmetry, similar to that of pristine graphene. Also, $H_{\text {eff }}$ is invariant under the space-time inversion, $T I: H_{\text {eff }}=\sigma_{x} H_{\text {eff }}^{*} \sigma_{x}$, where $\sigma_{x}$ is the Pauli matrix. Therefore, the band crossing of $\pi$ and $\pi^{*}$ states exists at the Fermi level[52], but different from the pristine graphene it is not at the $K$ point[53]. This fact is in coincidence with the results of the first-principles calculations. By analyzing the effective Hamiltonian, we can find that only the matrix elements $\left[f_{1}(\mathbf{k})\right.$ and $\left.f_{2}(\mathbf{k})\right]$ are influenced by the attachment of groups, while the other matrix elements retain the original form. Therefore, with the decrease of the coverage, the influence of the functionalization groups is suppressed, and the electronic properties of d-CFG 
should converge to those of pristine graphene when the coverage is small enough. This also coincides with the observation from the first-principles calculations.

The quantum transport properties of the s-CFG, d-CFG and the pristine graphene are further calculated to demonstrate the intrinsic overall effects of the periodical covalent functionalization of graphene by using non-equilibrium Green's functions (NEGF) methods combined with the density functional theory (DFT) calculations in the generalized gradient approximation. Figure 5 shows the calculated current-voltage $(I-V)$ curves both for s-CFG and $\mathrm{d}-\mathrm{CFG}$ with the coverage of $0.20 \mathrm{nmol} / \mathrm{cm}^{2}$. Evidently, the s-CFG exhibits semiconducting behavior as the current is negligible when the bias voltage is smaller than $0.4 \mathrm{~V}$. On the other hand, a metallic transport behavior can be observed for the d-CFG, which is mainly contributed by the $\pi$ electrons of graphene sheet.[54] These observations suggest that d-CFG has great potential as conducting layer and s-CFG can be used as TBP sensor. Under the same voltage, the current of d-CFG with the coverage of $0.20 \mathrm{nmol} / \mathrm{cm}^{2}$ is about one third of that in the pristine graphene, and this ratio is in line with that of Fermi velocities between the d-CFG and pristine graphene (shown in Table 1). Therefore, the current in d-CFG could be flexibly tuned by changing the TBP coverage which links with the Fermi velocity in the quantum transport region. This provides an effective method to manipulate the metallicity of d-CFG, and reveals its promising potential in the future nanoelectronics.

The above analysis indicates that the metallicity of d-CFG mainly originates from $\pi$ electrons of graphene, while having little connection with the detailed structure of TBP groups. It is thus that one may expect a universal metallic behavior for graphene functionalized with other phenyl groups. Recent experiments have shown that nitrophenyl groups can be covalently bonded to the graphene basal plane, resulting in a superlattice microstructure of the functionalized graphene, as manifested by atomic force microscopy, transmission electron microscopy and selected area electron diffraction [28]. We thus further calculate the electronic properties of graphene with double-side attachment of nitrophenyl groups. A similar band structure to that of d-CFG functionalized with TBP is observed, but with a difference in the Fermi velocity of carriers[55]. Recent Raman spectroscopy and electric transport measurements by Huang et al.[26] showed that CFG functionalized with nitrophenyl groups exhibits good metallic behavior, with conductivity as high as that of exfoliated graphene. Since the samples were synthesized in the solution, [26] nitrophenyl groups can be attached to both sides of graphene sheet, resulting in a metallic behavior as predicted from our calcula- 
tions. In contrast, if the phenyl groups are chemically adsorbed on exfoliated graphene sheet supported by the substrate to lead to single-side attachment, the transport measurement showed a semiconducting behavior.[22, 38] The agreement between all of these experimental observations and our theoretical predictions indicates that the electronic properties of CFG are sensitive to the fabrication process and the chemical functionalized graphene with high conductivity can be achieved by controlling the coverage elaborately. This finding provides a flexible method to control the electronic properties of graphene via chemical functionalization.

\section{CONCLUSIONS}

The structural and electronic properties of graphene covalently functionalized by TBP groups are investigated using first-principles calculations. The s-CFG exhibits a quasilocalized VBS, which can open a band gap and induce ferromagnetism in graphene as expected. The quasi-localized VBS also activates the nearest-neighboring atoms next to attachment site, leading to the attachment of another TBP group on the opposite side of graphene. In the d-CFG, two bands ( $\pi$ and $\pi^{*}$ states) cross each other at the Fermi level and the electron-hole symmetry is preserved, which can be qualitatively understood via a tight-binding model Hamiltonian analysis. And the transport properties can be flexibly tuned by changing the TBP coverage. The quantum transport calculations show that the s-CFG exhibits a semiconducting behavior, whereas the d-CFG a metallic transport behavior, which are consistent with the band structure calculations. Since the two crossing bands have little connection with the detailed structure of TBP group, the metallic behavior can also occur in CFG functionalized by other phenyl groups, such as nitrophenyl groups, as confirmed by our further calculations. This may provide an explicit mechanism to resolve the controversy of recent conductivity measurements of graphene functionalized with phenyl groups. Our work also suggests that functionalized graphene can be assembled into various electronic devices by controlling the attachment of phenyl group, which may have potential applications as chemical sensor and conducting layer. 


\section{Acknowledgments}

We acknowledge the support of the Ministry of Science and Technology of China (Grant Nos. 2011CB606405 and 2011CB921901), the National Natural Science Foundation of China (Grant Nos 11074139, 11104155 and 51232005), and the Shenzhen Projects for Basic Research (Nos. JC201105201119A and JCYJ20120831165730910). AR acknowledges financial support from the European Research Council Advanced Grant DYNamo (ERC2010-AdG -Proposal No. 267374) Spanish Grants (FIS2011-65702-C02-01 and PIB2010US00652), Grupos Consolidados UPV/EHU del Gobierno Vasco and European Commission project CRONOS (280879-2 CRONOS CP-FP7).

[1] A. K. Geim and K. S. Novoselov, Nat. Mater., 2007, 6, 183-191.

[2] M. Allen, V. Tung and R. Kaner, Chem. Rev., 2010, 110, 132-145.

[3] M. S. Fuhrer, C. N. Lau and A. H. MacDonald, MRS Bull., 2010, 35, 289-295.

[4] Q. Yan, B. Huang, J. Yu, F. Zheng, J. Zang, J. Wu, B. -L. Gu, F. Liu and W. H. Duan, Nano Lett., 2007, 7, 1469-1473.

[5] K. I. Bolotin, K. J. Sikes, Z. Jiang, M. Klima, G. Fudenberg, J. Hone, P. Kim and H. L. Stormer, Solid State Commun., 2008, 146, 351-355.

[6] S. V. Morozov, K. S. Novoselov, M. I. Katsnelson, F. Schedin, D. C. Elias, J. A. Jaszczak and A. K. Geim, Phys. Rev. Lett., 2008, 100, 016602.

[7] J. Moser, A. Barreiro and A. Bachtold, Appl. Phys. Lett., 2007, 91, 163513.

[8] A. A. Balandin, S. Ghosh, W. Bao, I. Calizo, D. Teweldebrhan, F. Miao and C. N. Lau, Nano Lett., 2008, 8, 902-907.

[9] Y. Xu, X. B. Chen, J. S. Wang, B. L. Gu and W. H. Duan, Phys. Rev. B, 2010, 81, 195425.

[10] P. C. Chen, Y. C. Li, C. Si, J. Wu, J. Ihm and W. H. Duan, Appl. Phys. Lett., 2012, 101, 033105.

[11] C. Soldano, A. Mahmood and E. Dujardin, Carbon, 2010, 48, 2127-2150.

[12] J. Hass, W. A. de Heer and E. H. Conrad, J. Phys.: Condens. Matter, 2008, 20, 323202.

[13] J. Wintterlin and M. L. Bocquet, Surf. Sci., 2009, 603, 1841-1852.

[14] X. Cui, C. Z. Zhang, R. Hao and Y. L. Hou, Nanoscale. 2011, 3, 2118-2126. 
[15] J. M. Englert, J. Roehrl, C. D. Schmidt, R. Graupner, M. Hundhausen, F. Hauke and A. Hirsch, Adv. Mater., 2009, 21, 4265-4269.

[16] Y. Hernandez, V. Nicolosi, M. Lotya, F. M. Blighe, Z. Sun, S. De, I. T. McGovern, B. Holland, M. Byrne, Y. K. Gun'Ko and et. al. Nat. Nanotechnol., 2008, 3, 563-568.

[17] J. R. Lomeda, C. D. Doyle, D. V. Kosynkin, W. -F. Hwang and J. M. Tour, J. Am. Chem. Soc., 2008, 130, 16201-16206.

[18] M. Delamar, R. Hitmi, J. Pinson and J. M. Saveant, J. Am. Chem. Soc., 1992, 114, 5883-5884.

[19] Y. C. Liu and R. L. McCreery, J. Am. Chem. Soc., 1995, 117, 11254-11259.

[20] J. L. Bahr, J. Yang, D. V. Kosynkin, M. J. Bronikowski, R. E. Smalley and J. M. Tour, J. Am. Chem. Soc., 2001, 123, 6536-6542.

[21] M. Wu, X. Wu, Y. Gao and X. C. Zeng, Appl. Phys. Lett., 2009, 94, 223111.

[22] E. Bekyarova, M. E. Itkis, P. Ramesh, C. Berger, M. Sprinkle, W. A. de Heer and R. C. Haddon, J. Am. Chem. Soc., 2009, 131, 1336-1337.

[23] L. M. Zhang, J. Yu, M. M. Yang, Q. Xie, H. L. Peng and Z. F. Liu, Nat. Commun., 2013, 4, 1443.

[24] S. Niyogi, E. Bekyarova, M. E. Itkis, H. Zhang, K. Shepperd, J. Hicks, M. Sprinkle, C. Berger, C. N. Lau, W. A. de Heer, E. H. Conrad and R. C. Haddon, Nano Lett., 2010, 10, 4061-4066.

[25] H. Zhang, E. Bekyarova, J. -W. Huang, Z. Zhao, W. Z. Bao, F. L. Wang, R. C. Haddon and C. N. Lau, Nano Lett., 2011, 11, 4047-4051.

[26] P. Huang, H. R. Zhu, L. Jing, Y. L. Zhao and X. Y. Gao, ACS Nano, 2011, 5, 7945-7949.

[27] J. M. Englert, C. Dotzer, G. Yang, M. Schmid, C. Papp, J. M. Gottfried, H. -P. Steinrück, E. Spiecker, F. Hauke and A. Hirsch, Nat. Chem., 2011, 3, 279-286.

[28] H. Zhu, P. Huang, L. Jing, T. Zuo, Y. L. Zhao and X. Y. Gao, J. Mater. Chem., 2012, 22, 2063-2068.

[29] T. O. Wehling, A. V. Balatsky, M. I. Katsnelson, A. I. Lichtenstein, K. Scharnberg and R. Wiesendanger, Phys. Rev. B, 2007, 75, 125425.

[30] J. M. García-Lastra, K. S. Thygesen, M. Strange and A. Rubio, Phys. Rev. Lett., 2008, 101, 236806.

[31] C. Bena and S. A. Kivelson, Phys. Rev. B, 2005, 72, 125432.

[32] V. K. Dugaev, V. I. Litvinov and J. Barnas, Phys. Rev. B, 2006, 74, 224438.

[33] O. V. Yazyev and L. Helm, Phys. Rev. B, 2007, 75, 125408. 
[34] B. Uchoa, V. N. Kotov, N. M. R. Peres and A. H. Castro Neto, Phys. Rev. Lett., 2008, 101, 026805 .

[35] P. Lu, R. Zhou, W. Guo and X. C. Zeng, J. Phys. Chem. C, 2012, 116, 13722-13730.

[36] J. M. García-Lastra, Phys. Rev. B, 2010, 82, 235418.

[37] A. R. Muniz and D. Maroudas, J. Appl. Phys., 2012, 111, 043513.

[38] A. Sinitskii, A. Dimiev, D. A. Corley, A. A. Fursina, D. V. Kosynkin and J. M. Tour, ACS Nano, 2010, 4, 1949-1954.

[39] J. Hong, S. Niyogi, E. Bekyarova, M. E. Itkis, P. Ramesh, N. Amos, D. Litvinov, C. Berger, W. A. de Heer, S. Khizroev and R. C. Haddon, Small, 2011, 7, 1175-1180.

[40] J. Hong, E. Bekyarova, P. Liang, W. A. de Heer, D. C. Haddon and S. Khizroev, Sci. Rep., $2012, \mathbf{2}, 624$.

[41] G. Kresse and J. Furthmüller, Phys. Rev. B, 1996, 54, 11169-11186.

[42] G. Kresse and D. Joubert, Phys. Rev. B, 1999, 59, 1758-1775.

[43] J. Perdew, K. Burke and M. Ernzerhof, Phys. Rev. Lett., 1996, 77, 3865-3868.

[44] Ž. Šljivančanin, M. Andersen, L. Hornekær and B. Hammer, Phys. Rev. B, 2011, 83, 205426.

[45] J. Taylor, H. Guo and J. Wang, Phys. Rev. B, 2001, 63, 245407.

[46] D. W. Boukhvalov and M. I. Katsnelson, J. Phys.: Condens. Matter, 2009, 21, 344205.

[47] W. X. Li, C. Stampfl and M. Scheffler, Phys. Rev. B, 2002, 65, 075407.

[48] D. Jiang, B. G. Sumpter and S. Dai, J. Phys. Chem. B, 2006, 110, 23628-23632.

[49] D. A. Siegel, C. H. Parka, C. Hwangb, J. Deslippea, A. F. Fedorovc, S. G. Louie and A. Lanzaraa, Proc. Natl. Acad. Sci. U.S.A., 2011, 108, 11365.

[50] C. Attaccalite and A. Rubio, Phys. Status Solidi B, 2009, 246, 2523-2526.

[51] P. A. Lödin, J. Chem. Phys., 1951, 19, 1396.M

[52] J. L. Mañes, F. Guinea and M. A. Vozmediano, Phys. Rev. B, 2007, 75, 155424.

[53] The determinant of $H_{\text {eff }}$ is the product of its eigenvalues. So the solution of the equation $\operatorname{Det}\left[H_{\mathrm{eff}}\right]=0$ determines the band crossing point around Fermi level. In this case, the solution exists and depends on the spectific parameters. So the band crossing here can be observed and is not at the $\mathrm{K}$ point, which is consistent with DFT calculations.

[54] It should be noted that the electron-phonon (el-ph) coupling might play an important role in the transport properties of some systems. The el-ph coupling, however, will not change the semiconducting transport behavior of s-CFG with a calculated transport gap of $0.8 \mathrm{eV}$, since 
the magnitude of reducing gap by el-ph coupling is about tens of $\mathrm{meV}$ for carbon materials [V. Perebeinos, J. Tersoff, and P. Avouris, Phys. Rev. Lett. 2005, 94, 086802]. Moreover, the nature of metallic transport of d-CFG will not be changed by the el-ph coupling, which only leads to a renormalization of the Fermi velocity and electron mobility for graphene [D. A. Siegel et al., PNAS, 2011, 108, 11365; A. Akturk and N. Goldsman, J. App. Phys., 2008, 103, 053702.].

[55] The electronic property of d-CFG attached by nitrophenyl groups with $2 \times 2$ supercell is also investigated. However, a small $2 \times 2$ supercell (corresponding to high coverage of functional groups), is not suitable for d-CFG since there is no enough space to accompany the closeby methyl groups which belong to different TBP groups. In this case, owing to the small size of the supercell, the attachment of nitrophenyl groups makes the planer graphene sheet distorted strongly and the $s p^{2}$ bonding among graphene carbon atoms without nitrophenyl group attachment cannot be kept. So this distortion can break the symmetry of graphene lattice and strongly affect the electronic properties, resulting in a small band gap (around 0.1 $\mathrm{eV})$. 
(a)

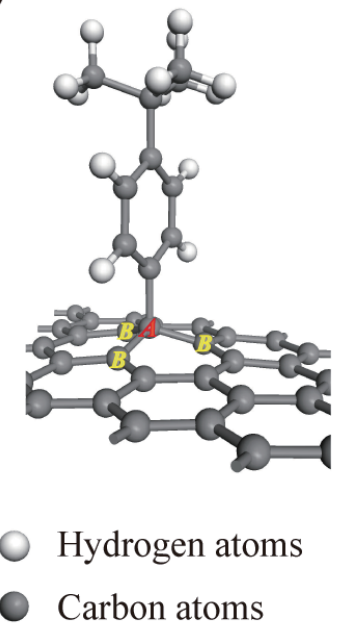

(b)

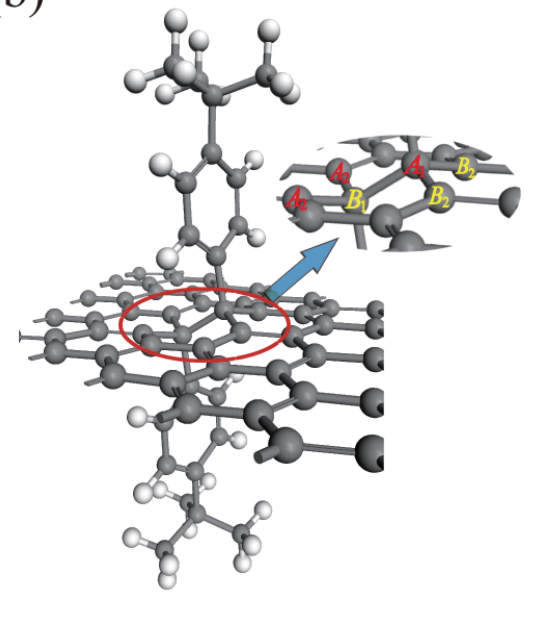

FIG. 1: Optimized geometrical structures of (a) single-side functionalized graphene by TBP groups (s-CFG) and (b) double-side functionalized graphene by TBP groups (d-CFG). The graphene carbon atoms marked A and B belong to different sublattices. 


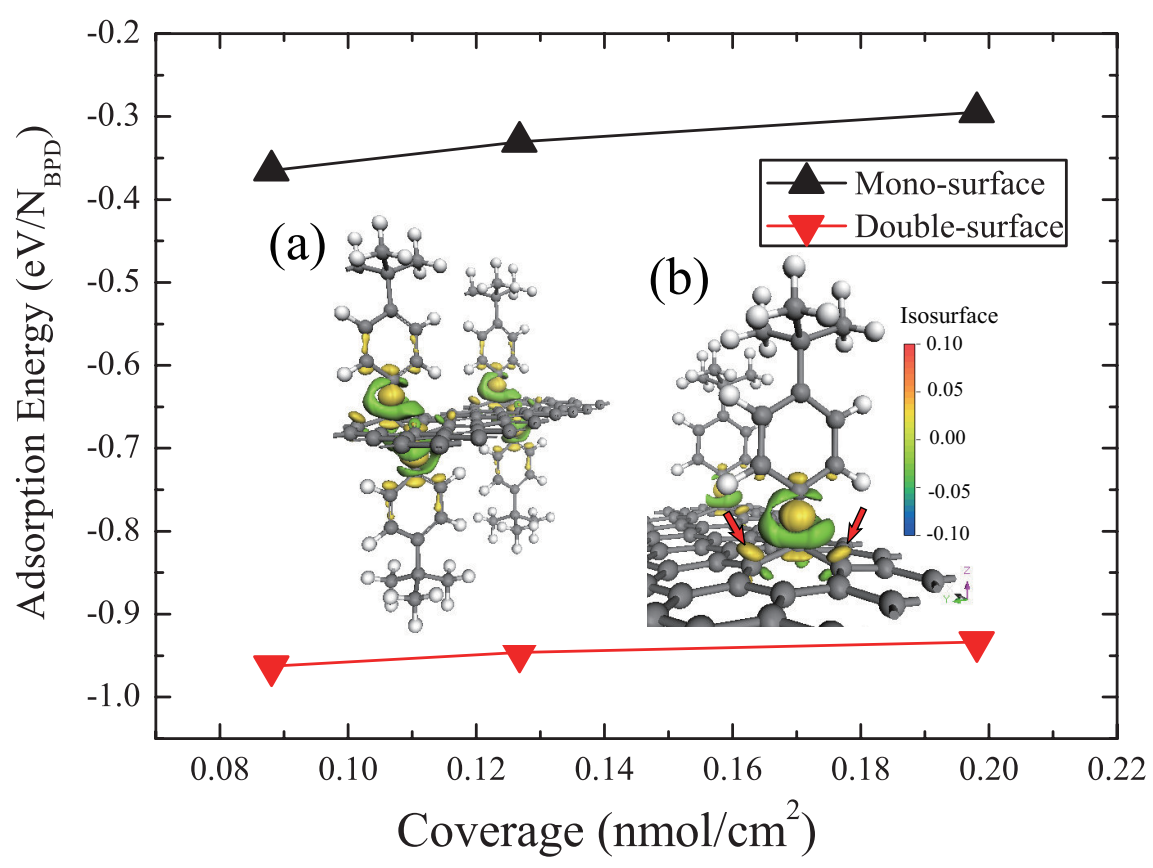

FIG. 2: Calculated adsorption energy per TBP group for different surface coverage and charge density difference (in units of e/ $\AA^{3}$ ) for (a) s-CFG and (b) d-CFG. The red arrows indicate the nearest-neighboring carbon atoms of the attachment site. The adsorption energy $E_{\text {ad }}$ is defined as $E_{\text {ad }}=\left(E_{\text {graphene }+n \mathrm{TBP}}-E_{\text {graphene }}-n E_{\mathrm{TBP}}\right) / n$, where $n$ is the number of TBP groups. 


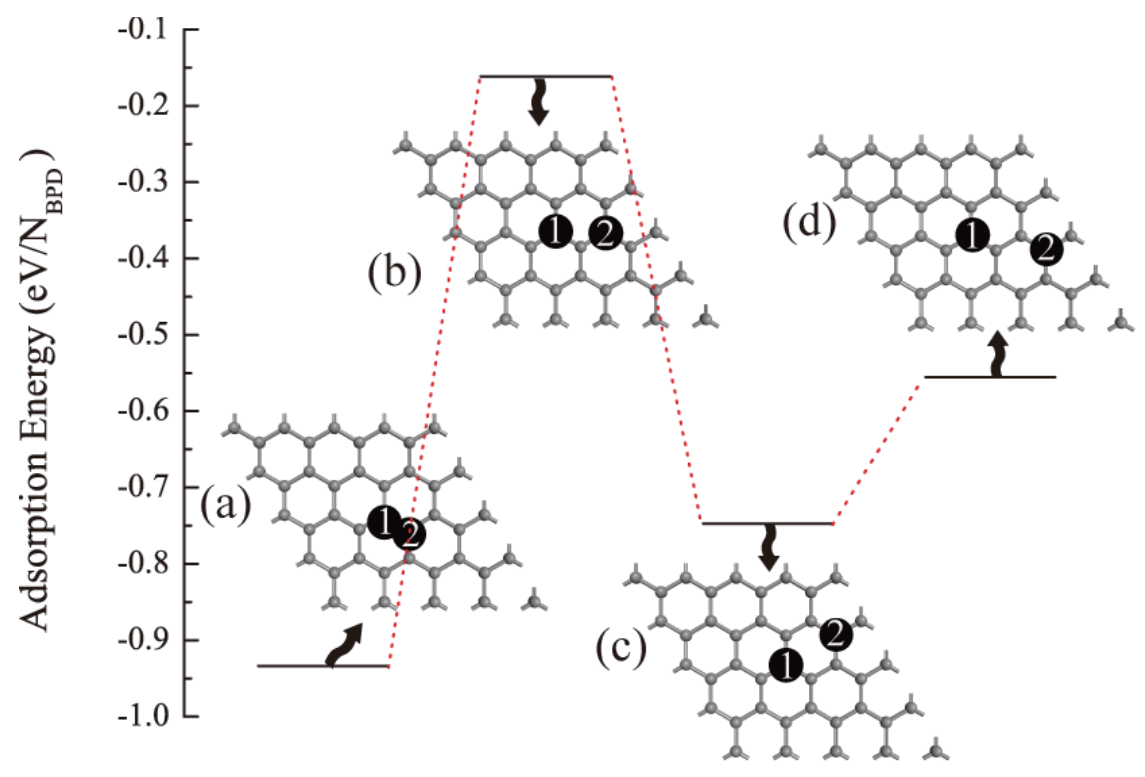

FIG. 3: Adsorption energies for attaching the second TBP group on the opposite side of graphene sheet in the $4 \times 4$ supercell model. Four different configurations (a-d: ortho, meta, para, and neighboring ring) of d-CFG are considered, where black circles with numbers 1 and 2 represent the sites for the first and second TBP attachments, respectively. 
(a)

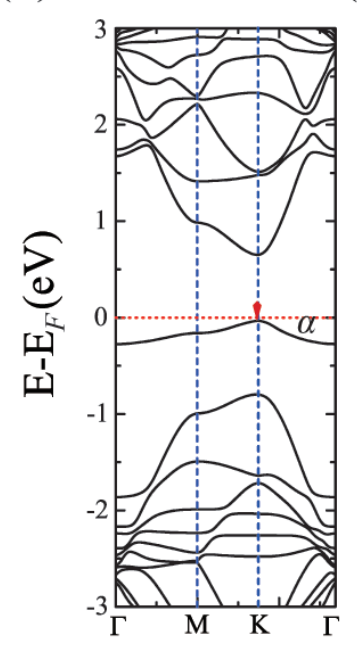

(b)

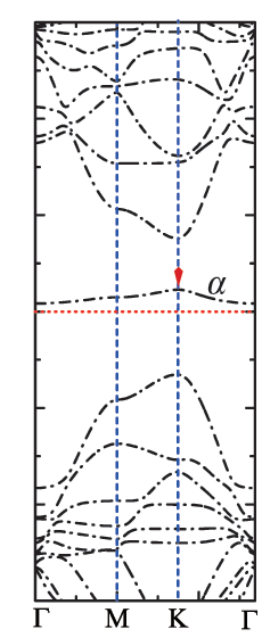

(c)

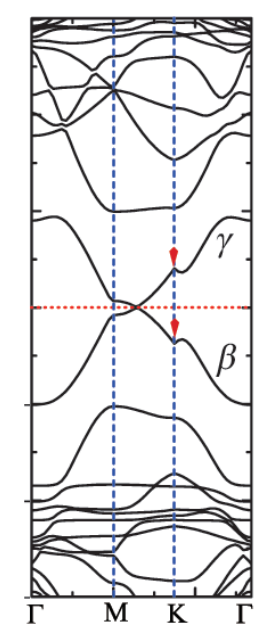

(d)

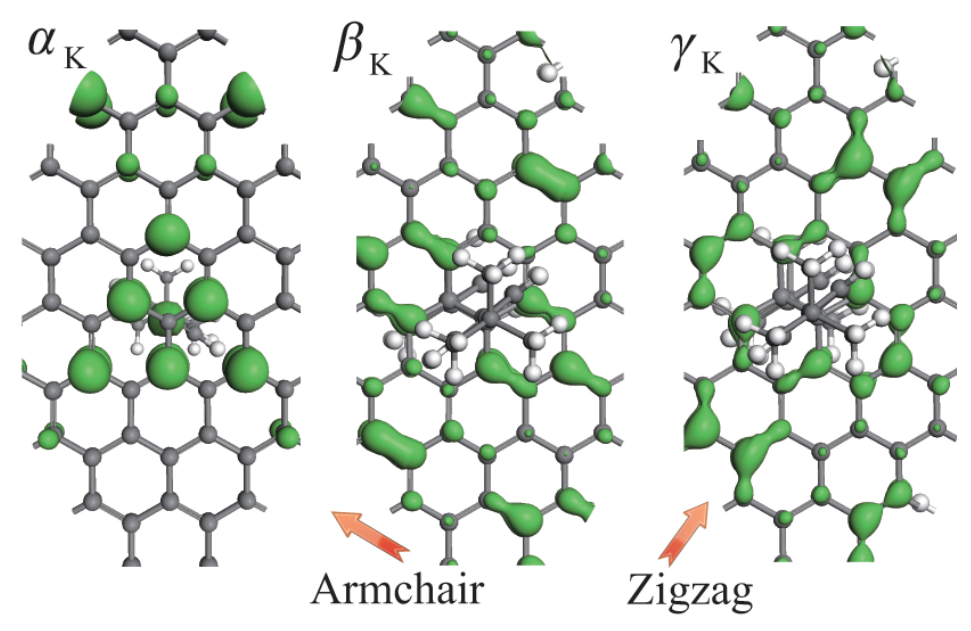

FIG. 4: (a) Majority-spin and (b) minority-spin band structures of s-CFG with the coverage of $0.20 \mathrm{nmol} / \mathrm{cm}^{2}$. (c) Band structure of d-CFG with the coverage of $0.20 \mathrm{nmol} / \mathrm{cm}^{2}$. Fermi levels are set to zero. (d) Isosurfaces of charge density $\left(0.03 \mathrm{e} / \AA^{3}\right)$ of the $\alpha, \beta$ and $\gamma$ bands at the $\mathrm{K}$ point. 


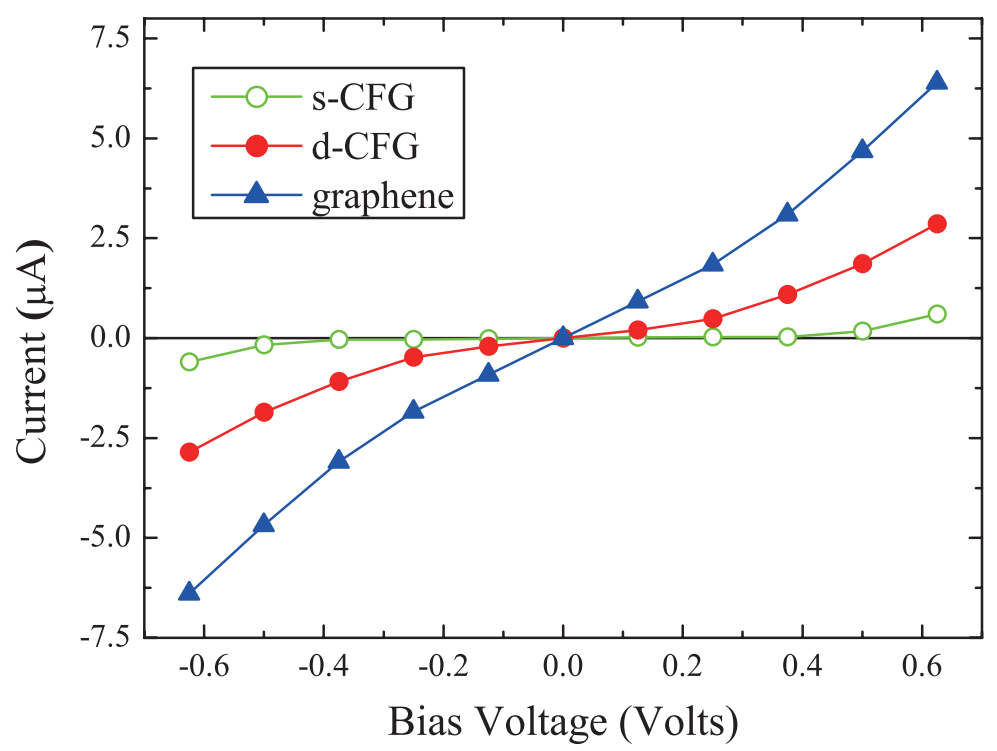

FIG. 5: (Color online) Current-voltage characteristics of s-CFG, d-CFG and the pristine graphene. The length of the scattering region is $\sim 9 \mathrm{~nm}$. 


\section{TABLE OF CONTENTS (TOC)}
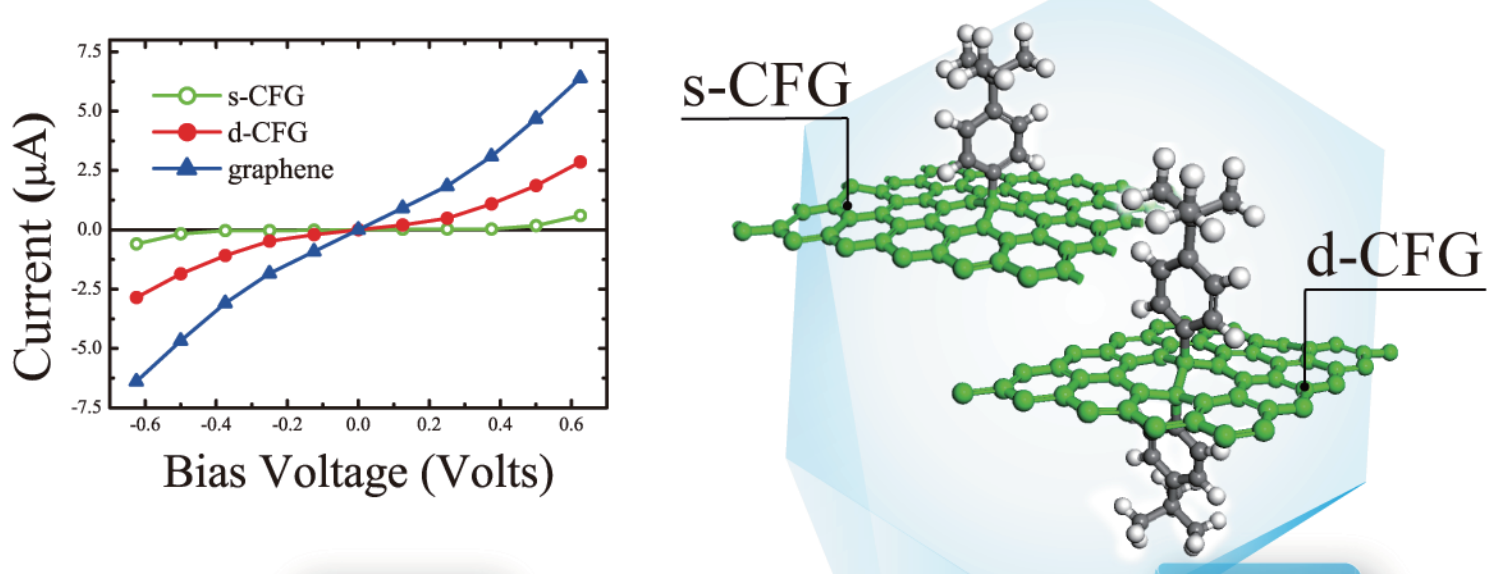

Graphene

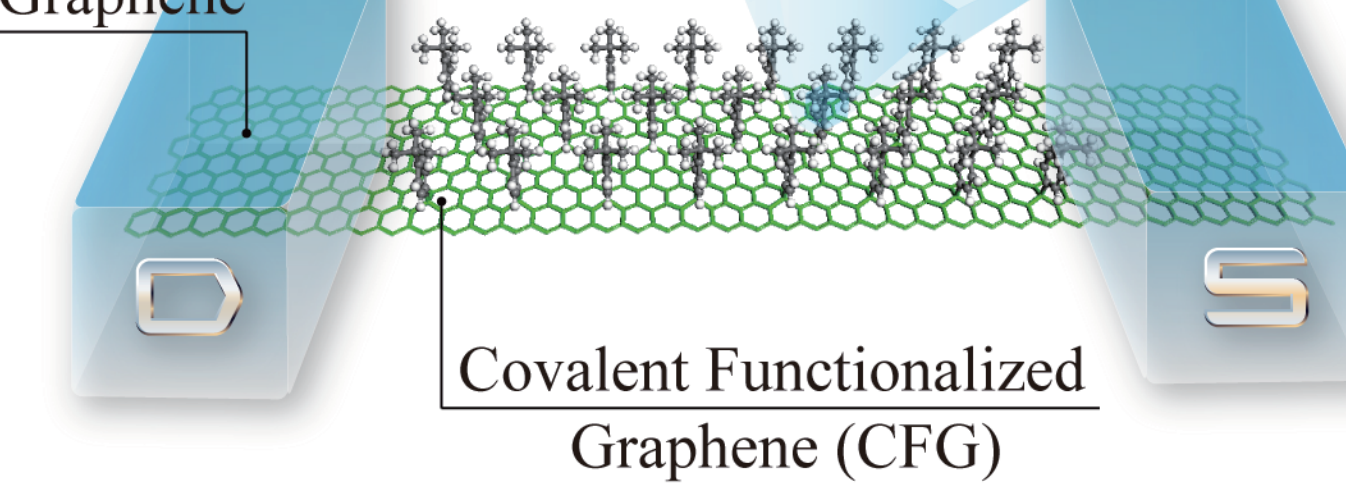

